\title{
A High-Linearity Digital-to-Time Converter \\ Technique: Constant-Slope Charging
}

\author{
Jiayoon Zhiyu Ru ${ }^{1,2}$, Claudia Palattella ${ }^{1}$, Paul Geraedts ${ }^{1,3}$, Eric Klumperink ${ }^{1}$, Bram Nauta ${ }^{1}$ \\ ${ }^{1}$ University of Twente, Enschede, Netherlands \\ ${ }^{2}$ now with Qualcomm ${ }^{3}$ now with Teledyne Dalsa \\ zhiyu.ru@gmail.com
}

\begin{abstract}
A digital-to-time converter (DTC) controls time delay by a digital code, which is useful, for example, in a sampling oscilloscope, fractional-N PLL, or timeinterleaved ADC. This paper proposes constant-slope charging as a method to realize a DTC with intrinsically better integral non-linearity (INL) compared to the popular variable-slope method. The proposed DTC chip realized in $65 \mathrm{~nm}$ CMOS consists of a voltage-controlled variable-delay element (DTC-core) driven by a 10-bit digital-toanalog converter. Measurements with a $55 \mathrm{MHz}$ crystal clock demonstrate a full-scale delay programmable from 19 ps to 189 ps with a resolution from $19 f$ s to $185 \mathrm{fs}$. As available oscilloscopes are not good enough to reliably measure such high timing resolution, a frequency-domain method has been developed that modulates a DTC edge and derives INL from spur strength. An INL of $0.17 \%$ at 189 ps full-scale delay and $0.34 \%$ at 19ps are measured, representing 8-9 bit effective INL-limited resolution. Output rms jitter is better than $210 \mathrm{fs}$ limited by the test setup, while the DTC consumes $1.8 \mathrm{~mW}$.

Index Terms - digital-to-time converter, DTC, integral nonlinearity, INL, phaselocked loop, PLL, constant slope, variable slope, delay, variable delay, delay measurement.
\end{abstract}




\section{INTRODUCTION}

Time delay is often defined as the time difference between the threshold-crossing points of two clock edges. If delay is programmable by a digital code, a digital-to-time converter (DTC) results. It is a basic building block suitable for several applications, e.g. fractional-N phase-locked loops (PLL) [1]-[4], (sub-)sampling oscilloscopes [5][6], automatic test equipment (ATE) [7], direct digital frequency synthesis (DDFS) [8], polar transmitter [9], radar [10], phased-array system [11], and time-interleaved ADC timing calibrations [12]. This paper aims at improving the time resolution and linearity of a DTC. A nominal full-scale delay in the order of 100ps is targeted with fine delay steps of less than $100 \mathrm{fs}$.

The basic element of a DTC is a variable-delay element, and there are different ways to implement delay in CMOS. A distributed circuit such as an ideal transmission line can theoretically provide true time delay while keeping the waveform undistorted. However, it requires unpractically long line length in CMOS technology (e.g. $100 \mathrm{ps} \times 2 \cdot 10^{8} \mathrm{~m} / \mathrm{s}=20 \mathrm{~mm}$ ). Moreover, as CMOS interconnect losses are high and frequency dependent, different amplitudes and waveforms result at different delay tap-points along a transmission line, which introduces zero-crossing variations when sensed by a comparator [13]. Lumped circuits such as all-pass filters can approximate a true time delay compactly [14][15] and maintain signal waveform, but noise and dynamic range are compromised.

If the waveform is not important and delayed clock generation is the purpose, digital circuits can be used. Minimum digital gate delays are on the order of 10ps in 65nm CMOS. However, if the difference between two gate delays is used, or if the gate delay is tunable, much smaller delay steps can be realized, for example in the order of $100 \mathrm{fs}$ as will be presented in this work. Although the absolute delay is still limited by the intrinsic gate delay, the relative delay steps can be much smaller. 
If delay tuning is linear, a high-linearity DTC can be realized. A linear DTC is favored, as calibration of only two points is sufficient, in contrast to a non-linear DTC that require multipoint calibration [16]. To characterize linearity, integral nonlinearity (INL) is an important metric for a DTC, similar to digital-to-analog converters (DAC). Non-zero DTC INL limits the achievable spur level in fractional-N PLLs [1]-[3] [16] and the timing accuracy in sampling oscilloscopes.

A DTC often exploits a voltage ramp generated by a current source charging a capacitor, and a comparator with threshold voltage $V_{d}$ defining a time delay $t_{d}$ (see Fig. 1). Switched capacitors [1][2][4] or switched current sources [3][6][7] can be applied to program delay. These approaches produce a delay by varying the slope from one ramp to another, which we refer to as the variable-slope method (see Fig. 2a). Using this method, 300fs delay resolution has been achieved in [2]. However, high resolution does not necessarily mean high linearity. In this paper, we propose a constant-slope method in which all ramps ideally would have the same slope, in contrast to the variable-slope method (see Fig. 2b). To still realize variable delay, a variable start voltage is used which can linearly program delay. We will show that this method is intrinsically more linear, allowing for a more linear DTC than variable slope offers. Before we do this in the next section, we first briefly discuss related previous work.

In [17], the nonlinearity of the variable-slope method was observed but not explained. The use of a high-gain comparator to improve INL was proposed in [17], but no measurement results were reported.

In [5] , delay is controlled by tuning the threshold voltage of a comparator, which would result in linear delay control if the slope of the ramp does not change over the threshold tuning range. Practically this is challenging, as the current produced by a current source as shown in Fig. 1 depends on the voltage across it, and hence on the capacitor voltage $\mathrm{V}_{\mathrm{C}}$. Moreover, the 
comparator in [5] works at varying common-mode voltage, leading to a variable speed of the comparator, i.e. an extra INL source.

Another way to realize variable delay is by phase interpolation, which can be implemented using current sources [18][19], resistors [20][21] or delay lines [22]. The basic concept of interpolation and example waveforms are shown in Fig. 3, where the middle parts are constant-slope, assuming $\mathrm{V}_{\mathrm{A}}$ and $\mathrm{V}_{\mathrm{B}}$ have the same slope. However, phase interpolation is functionally different as it requires two edges to be present, between which it can place a new edge. In contrast, this work aims to produce a delayed edge after one incoming critical edge that triggers one charging process.

The main new contributions of this paper are threefold: 1) a concept to define a constantslope method and to identify its fundamental advantages in terms of INL compared to a variable-slope method; 2) a new circuit topology in which the start voltage controls the delay of only one critical edge, leading to high linearity and low jitter; 3) measurement results demonstrating a fine resolution and a small INL, for which a new measurement method was devised.

This paper is organized as follows: section II explains the constant-slope method and its advantage in linearity; section III describes the design of a DTC circuit using this method; section IV presents measurements and section V conclusions.

\section{CONSTANT-SLOPE Method}

\section{A. Constant-Slope Ramp Generation}

To generate a voltage ramp with a controlled slope $(S=\Delta v / \Delta t)$, often a current is used to charge a capacitor as shown in Fig. 1 , where $S=I / C$. The delay time $\left(\mathrm{t}_{\mathrm{d}}\right)$ of this ramp from zero voltage to the voltage $V_{d}$ is: 


$$
t_{d}=V_{d} / S
$$

As shown by (1), if we want one variable to control delay, we can either vary the slope $S$ ("variable slope") at fixed voltage $\mathrm{V}_{\mathrm{d}}$, or keep the slope fixed and vary voltage $\mathrm{V}_{\mathrm{d}}$. In practice, however, a single ramp often has a changing slope as shown in Fig. 1(b), therefore varying $V_{d}$ does not always give a linearly-controlled delay.

Instead, we can vary the start-voltage $V_{\text {st }}$ as shown in Fig. 4 between 0 and $V_{\text {st,max }}$. To generate a linearly-controlled delay, it is sufficient if the part below $\mathrm{V}_{\mathrm{st} \text {,max }}$ is constant-slope, while for the part above $\mathrm{V}_{\text {st,max }}$ it suffices to have a constant-shape ${ }^{1}$. As the trajectory above $\mathrm{V}_{\text {st,max }}$ is shared for all ramps and adds a fixed amount of delay, it does not affect the linearity of the delay control function. Similarly, the same ramp start-up behavior between $t_{0}$ and $t_{1}$ adds a delay offset to all ramps which does not hurt linearity either.

A constant-shape above $V_{\text {st,max }}$ ensures that at different $V_{d}$, the delay $\tau$ between two ramps keeps constant, and it also renders INL benefits as described below.

\section{B. Advantage of Constant-Slope Method on INL}

We will use simple models to gain intuitive understanding. The delay function in Fig. 2 contains two distinct actions: 1) ramp generation and 2) threshold comparison. The ramp generation produces a ramp with controlled slope, while the threshold comparison defines a decision threshold $V_{d}$ and produces an output edge when crossing the threshold. The variableslope-induced INL comes from the behavior of a practical comparator.

One source of delay INL is the comparator bandwidth limit, which can be modeled by adding an $R C$ network at the comparator output. It can be derived that, in case of an input ramp signal, the propagation delay of an $R C$ network of any order contains nonlinear functions of the input ramp time [23][24], e.g. exponential and logarithmic functions.

\footnotetext{
${ }^{1}$ Being constant-shape between two ramps is equivalent to having the same (local) slope at equal ramp voltage. This property allows for an alternative but important interpretation of the name "constant slope”, namely that the (local) slope is constant when comparing ramps of different delay settings at equal ramp voltage.
} 
Assuming linear ramp generation, the ramp time would vary linearly with code, however the propagation delay would vary nonlinearly with code due to its nonlinear function versus input ramp time in an RC network. Since poles are ubiquitous in circuits related to parasitic resistance and capacitance in transistors and interconnects, this is a source of INL in a DTC.

Another source of delay INL can be explained by the example of using an inverter as comparator. The nonlinear relationship between an inverter's delay and its input ramp time has been modeled in [25] by equations using empirical parameters obtained from simulation fitting. Three operating modes were distinguished in an inverter's response to an input ramp signal: overshoot recovery, short circuit, and output discharge [25]. During overshoot recovery, the output recovers from overshoot due to an initial input switching event; the shortcircuit mode occurs when both the PMOS and NMOS conduct (but with different currents so non-zero output slope), resulting in "short circuiting" of the supply; the output-discharge mode refers to the mode with only the NMOS on. For different input slopes, the three modes contribute differently to the output transition time, which is another source of INL in a DTC. This mechanism applies to any comparator that passes through different operating modes during its input and output transitions.

To avoid the INL error associated with variable slope, we propose the constant-slope method in which the ramps keep a constant shape above $\mathrm{V}_{\text {st,max }}$, whose effect on a comparator is modeled in Fig. 5. Two rising ramps $a$ and $b$ at the input of the comparator have different start voltages but the same shape above $\mathrm{V}_{\text {st }}$. A delay difference $\tau$ is sensed by the comparator to produce two corresponding falling edges at the output.

Actually, the output of a practical comparator responds to a range of input voltages and modelling it as a simple comparator with one exact threshold is somewhat simplistic. Instead of a threshold, it is perhaps better to talk about a "comparator input window”, for example between $V_{\text {tho }}$ and $V_{\text {th1 }}$ in Fig. 5. When the input voltage rises to $V_{\text {th0 }}$, the output voltage 
begins to change as the comparator starts to discharge the output node (a comparator with inverted output is assumed here).

If $\mathrm{V}_{\text {st }}<\mathrm{V}_{\text {th0 }}$, the start-voltage part of the ramp does not affect the comparator response. Only the part of the ramp within the input window affects the output. In Fig. 5, if the ramps $a$ and $b$ have the same shape between $\mathrm{V}_{\text {th0 }}$ and $\mathrm{V}_{\text {th1 }}$, they evoke the same response at the output. Hence their propagation delays through the comparator are equal, and both edges would also have a constant shape at the output of the comparator, no matter what the bandwidth is. Therefore the time shift between two edges at the output is the same as at the input, and there is ideally no error. Furthermore, unlike the variable-slope case, because all edges have the same shape also at the output, INL errors are also avoided in later stages, e.g. buffer stages that further steepen the output edge.

If a comparator passes through different operating modes during its transition as modeled in [25], the constant-slope method still renders benefits in INL. The reason is again that, apart from a different start voltage, both ramps in Fig. 5 have the same shape within the critical input window of the comparator whose response to both ramps is then very similar. Hence each operating mode, e.g. the short circuit or output discharge modes discussed above, renders the same contribution to the output edge for ramp $a$ and $b$. The overshoot depends on the start-voltage level, however as long as the start voltages are well below the "comparator input window” then the overshoot at the output can recover before the input reaches that window, so its contribution to delay can be negligible.

To intuitively summarize, any (correlated) differences between ramps at the comparator input tend to cause INL error. In the constant-slope method, the start voltage is different, other than which all ramps have the same shape. So the comparator does not introduce INL as long as it hardly reacts to the start-voltage part from 0 to $\mathrm{V}_{\text {st,max }}$. In variable-slope method, the 
slope is different among all ramps in the comparator input window, so it may introduce significant INL through comparator as shown in the next sub-section.

\section{Simulation}

The setup in Fig. 6 is used to simulate the INL for an ideal input ramp signal. Circuit simulations were done in a $65 \mathrm{~nm}$ CMOS technology at $1.2 \mathrm{~V}$ supply. In clocking applications, a simple inverter is often used to implement the threshold comparison and can also act as a buffer to produce a steep output edge. In Fig. 6, after the first inverter acting as a comparator, a four-stage inverter chain of identical inverters is applied to boost the slope to values close to the technology-dependent speed limit (e.g. 50 100GV/s in 65nm). This is for instance desired in sampler or phase detector applications, to precisely define the timing.

To simulate the variable-slope case, 100-300ps rise time from GND to VDD was used, ideally resulting in 50-150ps delay at half-VDD comparator threshold, so 100ps delay-control range. The simulation results are shown in Fig. 7 which have an actual range of 110ps, as the inverter threshold is not exactly half-VDD. 100 steps were taken over the whole delay range and a maximum INL of $1.4 p s$ is found, which is in the same order of magnitude as results found in literature [1][2][7].

For the constant-slope case a variable start-voltage range from 0 to $0.2 \mathrm{~V}$ was used (motivated later in this paper). Mapping this range to a 100ps delay, the rise time is 600ps from $0 \mathrm{~V}$ to $1.2 \mathrm{VDD}$ and 500ps from $0.2 \mathrm{~V}$ to $1.2 \mathrm{VDD}$. Simulation results in Fig. 8 show a maximum INL of only 15fs, about two orders of magnitude lower than for variable slope. This clearly demonstrates the INL advantage of the constant-slope method, via the example of a simple inverter as a comparator.

In summary, the proposed constant-slope method modulates delay by changing the start voltage while keeping the critical threshold part of a ramp unaltered. Thus all ramps have the 
same shape in the "comparator input window", leading to the same propagation delay through a comparator or buffer stage, which minimizes INL errors.

\section{DTC DEMONSTRATOR CHIP DESIGN}

To demonstrate good INL in practice, a DTC chip has been implemented in 65nm CMOS with $1.2 \mathrm{~V}$ supply. Fig. 9 shows the block diagram of the chip. The DTC-core is a voltagecontrolled variable-delay element, which consists of a low-noise buffer (LNB), a ramp generator, and a threshold comparator. The amount of delay is controllable by a 10-bit DAC. The DTC is driven by a sine-wave from a crystal oscillator (XO), and its output delivers a rectangular-wave clock with a variable delay. An inverting buffer with $50 \Omega$ output impedance drives the off-chip transmission line for measurements. We will discuss the design of the main blocks in the following subsections, and will also discuss INL error sources.

\section{A. DTC-core}

Fig. 10 shows the schematic of the DTC-core. Its sub-blocks are discussed below.

\section{1) Low-Noise Buffer}

The low-noise buffer converts a sine-wave into a rectangular-wave with low added jitter. The noise of the first stage is critical given the relatively low slew-rate of a sine-wave from a $55 \mathrm{MHz}$ crystal. As only one edge is critical, big NMOS transistors are used for low noise while the PMOS is small and is controlled by its driver in such a way that simultaneous conduction of the PMOS and NMOS is reduced [26]. The driver (D1) of the PMOS is shown in Fig. 11, which produces a small duty cycle therefore low supply "short-circuit" current. The big “poor man’s cascode” NMOS in Fig. 11 (sized 2000/0.06) helps to boost the output impedance, without requiring a dedicated bias voltage. Therefore the voltage gain around the zero-crossing points of the input rising edges is increased and so is the falling-edge steepness at the output node $X$, which benefits timing jitter. 


\section{2) Ramp Generator}

The core of the ramp generator in Fig. 10 consists of $\mathrm{M}_{\mathrm{P} 1}-\mathrm{M}_{\mathrm{P} 3}$ that produce the charging current to capacitor $C_{0}$ to realize a ramp voltage. In every cycle of the DTC, node $Y$ is first reset to GND via $\mathrm{M}_{\mathrm{N} 2}$, then pre-charged to $\mathrm{V}_{\mathrm{st}}$ via $\mathrm{M}_{\mathrm{N} 1}\left(V_{s t}=I_{D A C} \cdot R_{D A C}\right)$, after which a ramp takes place. The timing of the reset and pre-charge of $\mathrm{C}_{0}$ and the ramp is controlled by three signals which are all derived from the same input: $V_{\text {res }}, V_{\text {prech }}$ and $C L K_{\text {in }}$. Produced by LNB with two buffers, CLK $_{\text {in }}$ delivers the critical edge that activates $\mathrm{M}_{\mathrm{P} 1}$ to start the ramp. The driver D2 producing $\mathrm{V}_{\text {prech }}$ is the same as D1 shown in Fig. 11. The pulse generator producing $\mathrm{V}_{\text {res }}$ is made of an AND gate with two inputs whose delay difference defines the pulse width as shown in Fig. 12.

$\mathrm{M}_{\mathrm{P} 2}$ and $\mathrm{M}_{\mathrm{P} 3}$ form a current mirror with 10:1 ratio to create a charging current, derived from an external bias current $I_{b}$ for flexibility. The charging current can be up to a few $\mathrm{mA}$, but the average current consumption is on the order of a few hundred $\mathrm{uA}$ because $\mathrm{M}_{\mathrm{P} 2}$ only draws current during the ramp, which is only a small fraction of the clock period. A $6 \mathrm{pF}$ capacitor to VDD helps keep the gate voltage of $\mathrm{M}_{\mathrm{P} 2}$ stable and so its current. Then the different start voltages $\mathrm{V}_{\mathrm{st}}$ at node $Y$ have much reduced effect on $\mathrm{M}_{\mathrm{P} 3}$ and $\mathrm{I}_{\mathrm{b}} \cdot \mathrm{M}_{\mathrm{P} 1}$ acts as switch that starts the ramp, but also as cascode transistor to improve the output resistance of the current source $\mathrm{M}_{\mathrm{P} 2}$, and hence the linearity of the ramp voltage.

As the constant-slope method minimizes the INL associated with comparator, the remaining INL contribution is in ramp generation, which can be minimized by design.

Because delay is defined as $\Delta t=\Delta v /(I / C)$, for a linear delay, it is important to realize a constant current source and capacitance, during the first part of the ramp that defines delay via different $\mathrm{V}_{\text {st }}$ values. At a $1.2 \mathrm{~V}$ supply, this requirement limits the $\mathrm{V}_{\text {st }}$ in our circuit from GND up to about $V_{\text {st,max }}=200 \mathrm{mV}$, where $\mathrm{M}_{\mathrm{P} 1}$ still remains well in saturation and acts well as cascode. When a ramp goes beyond $\mathrm{V}_{\mathrm{st}, \max }, \mathrm{M}_{\mathrm{P} 1}$ would gradually enter the triode region, 
however this does not cause INL, because it is a common effect, i.e. the same waveform is shared for all ramps beyond $\mathrm{V}_{\text {st,max }}$.

The linear poly resistor $R_{D A C}$ defines $V_{\text {st }}$ based on current $I_{D A C}$. The nonlinear switch resistance $R_{\text {on }}$ of $M_{N 1}$ has negligible contribution to INL if $R_{D A C}>>R_{o n}$, which can be understood by applying Thévenin's theorem where $\mathrm{R}_{\mathrm{DAC}}$ and $\mathrm{R}_{\mathrm{on}}$ are in series. The linear metal capacitor $\mathrm{C}_{0}$ in parallel to the nonlinear parasitic capacitance at node $Y$ defines the slope of the voltage ramp. This improves linearity as the combined capacitance is less dependent on voltage.

Given a supply voltage, the usable start-voltage range is limited. If the voltage range is fixed, to achieve a larger delay requires a lower slope (i.e. slower ramp), posting a delay-jitter trade-off.

\section{3) Threshold Comparator}

The threshold comparator uses a simple inverter to sense the ramp voltage created at node $Y$. The nominal threshold voltage of the inverter was designed to be around half-VDD ( 600mV), which is much larger than the $200 \mathrm{mV}$ maximum $\mathrm{V}_{\text {st }}$. Buffer B3 (two scaled-up inverters) steepens the output edges.

\section{B. Digital-to-Analog Converter}

To save design time, an existing 10-bit current-steering DAC IP-block is co-integrated on the same chip. The segmented DAC is divided into two sub-DACs, a 5-bit binary-weighted sub-DAC for LSBs and a 5-bit unary-weighted sub-DAC for MSBs. For its performance, we rely on the specification datasheet, which however is not very detailed. Hence, we resort to calculations and estimations to derive some of the specifications.

The DAC is specified to operate at $2.5 \mathrm{~V}$ supply, but it can also operate at $1.2 \mathrm{~V}$ with a more limited output voltage range. The DAC specification indicates a maximum INL of \pm 2 LSB 
$( \pm 0.2 \%)$ at an output range of $0-800 \mathrm{mV}$ and a $400 \mathrm{MHz}$ speed. The INL should improve when only $0-200 \mathrm{mV}$ output range and $55 \mathrm{MHz}$ speed are used in this design.

The DAC noise is not found in the IP's datasheet. A first-order calculation was done assuming the thermal noise is dominant at a low switching speed of $55 \mathrm{MHz}$. For a currentsteering DAC, its current noise can be modelled as:

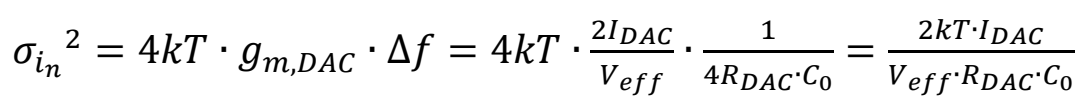

where $\Delta f$ is the equivalent DAC noise bandwidth defined as $1 /\left(4 R_{D A C} C_{0}\right)$ with $R_{D A C}$ and $C_{0}$ in Fig. 9, and $V_{e f f}$ is the overdrive voltage of the DAC current sources.

The DAC current noise is converted to voltage by $R_{D A C}$ and then produces timing jitter, which can be modelled as:

$$
\sigma_{\mathrm{t}}=\frac{\sigma_{i_{n}} \cdot R_{D A C}}{\Delta v / \Delta t}=\frac{1}{\Delta v / \Delta t} \cdot \sqrt{\frac{2 k T \cdot I_{D A C} \cdot R_{D A C}}{V_{e f f} \cdot C_{0}}}
$$

In our design, $\Delta v / \Delta t$ is equal to $200 \mathrm{mV} / 100 \mathrm{ps} ; I_{D A C}=0.87 \mathrm{~mA} ; R_{D A C}=200 \Omega ; C_{0}=1.3 \mathrm{pF}$; $k T=4.1 \mathrm{e}-21 \mathrm{~J}$. Even assuming a rather low $V_{\text {eff }}=150 \mathrm{mV}$, the result of (3) is $43 \mathrm{fs}$, i.e. $<0.5 \mathrm{LSB}$ for a 10-bit DAC and a 100ps full-scale delay. Note that this is at the maximum DAC output current, i.e. the worst-case noise.

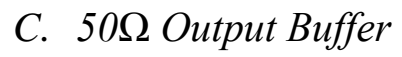

To be able to measure the DTC, an output buffer is designed which includes an inverter and an integrated $50 \Omega$ pull-up resistor (see Fig. 9). When connected to an off-chip cable and equipment with $50 \Omega$ to ground, the buffer output establishes a DC bias voltage nicely around half-VDD. The inverter is sized to provide around $0.6 \mathrm{~V}_{\mathrm{pp}}$ swing at the matched output so that the variation of inverter output resistance does not have much effect on the output impedance matching. 


\section{Error Sources}

A DTC is usually meant to produce a well-controlled amount of delay, however nonidealities such as noise, distortion, process-voltage-temperature (PVT) variations, and mismatches introduce timing errors.

For a switching circuit such as a DTC, the jitter is often lower with higher signal slope or larger transistor size and current. The jitter of the implemented DTC is dominated by the LNB due to the low sine-wave slope at $55 \mathrm{MHz}$ input.

Other than jitter, the timing error of a DTC can be divided into offset, linear and nonlinear errors. An offset error means a common delay shift to all delay steps while the relative delay from one step to another remains un-changed; a linear error means that all delay steps are scaled by the same ratio, i.e. full-scale delay changes but delay steps are still equal and there is no DNL or INL; nonlinear errors render a code dependent step size, leading to DNL/INL.

If the threshold voltage of an inverter varies over PVT, in a variable-slope method, this will cause offset and linear errors (so the full-scale) which can be seen in Fig. 2 by moving $V_{d}$ up and down, but potentially also different INL. For instance, INL in percentage changes if nonlinear errors scale differently than the full-scale delay does. On the other hand, a $\mathrm{V}_{\text {th-shift }}$ means that the inverter characteristic changes, which often also leads to INL change. Furthermore, a practical ramp is not a perfectly straight line and its slope is different at different voltage levels (see Fig. 1). When a ramp passes through an inverter for which PVT changes the threshold, the inverter sees a different slope, so the nonlinear effects change, and INL values change. Instead, for the constant-slope method only an offset in delay occurs, which can be seen in Fig. 2 and Fig. 5. It does not cause linear errors because the delay from one ramp to another is the same at any voltages. As explained in section II, it also intrinsically does not cause nonlinear errors by comparator no matter what the threshold is. 
Since the constant-slope method is robust to threshold comparison, the remaining error sources are mostly in delay generation.

In practice, the start-up behavior of a ramp is not instantaneous, but rather has an initial over-shoot due to capacitive coupling and a rounded start-up waveform because a charging current is not fully turned on instantaneously. Simulation indicates that this effect is largely independent of the start-voltage for the used $0-200 \mathrm{mV} \mathrm{V}_{\mathrm{st}}$-range. This mainly adds an offset to the delays.

A rising slope at node $Y$ in Fig. 10 can be written as

$$
S=\frac{10 \cdot I_{b}}{C_{0}}=\frac{V_{s t}}{\Delta t}=\frac{I_{D A C} \cdot R_{D A C}}{\Delta t}
$$

Eq. (4) can be re-arranged to derive the delay $\Delta \mathrm{t}$ :

$$
\Delta t=\frac{V_{s t}}{10 \cdot I_{b}} \cdot C_{0}=\frac{I_{D A C}}{10 \cdot I_{b}} \cdot R_{D A C} \cdot C_{0}
$$

Eq. (5) shows this delay is proportional to an RC time constant which is subject to PVT variations, introducing a linear error. The DAC current and the bias current can be derived from the same current source, so PVT variations are removed by taking the current ratio. However, any mismatch in current mirrors will introduce linear errors. Many applications will require the delay range to be aligned to another clock, e.g. a VCO period in a PLL, which will then also calibrate these linear errors.

The INL for the constant-slope design is related to the circuit nonlinearity caused by the varying $\mathrm{V}_{\mathrm{st}}$, including the nonlinearity of current source $\mathrm{M}_{\mathrm{P} 2}$, the nonlinearity of junction capacitance at node $Y$, and the nonlinearity of pre-charge switch $\mathrm{M}_{\mathrm{N} 1}$. The former two affect the $I / C$ ratio and therefore the slope, while the latter two affect the settling of $V_{\text {st }}$ due to nonlinearity in the RC time constant. Note that these nonlinearities have been largely reduced by measures discussed in section III.A.2), including cascode $\mathrm{M}_{\mathrm{P} 1}$, linear $\mathrm{C}_{0}$, and linear $\mathrm{R}_{\mathrm{DAC}}$. 
Furthermore, a relatively small $\mathrm{V}_{\mathrm{st}}$ range of $200 \mathrm{mV}$ helps limit these nonlinear effects; also, a $55 \mathrm{MHz}$ operating frequency gives enough settling time to reduce the settling error of $\mathrm{V}_{\mathrm{st}}$.

Another source of INL comes from the DAC. The mismatch of the DAC cells and the nonlinearity of its output impedance affect the DAC INL and therefore $\mathrm{V}_{\text {st }}$, directly translating to the delay INL as shown in (5). Note that the DAC INL similarly hurts the delay INL in a variable-slope method. This effect relates to the DAC design, and is not intrinsic nor distinctive for the constant or variable slope method.

\section{E. Simulation Results}

Using a PSP Model, the circuit in Fig. 10 has been simulated together with an ideal DAC producing 0 -to- $200 \mathrm{mV}$ as $\mathrm{V}_{\text {st }}$. The input of the LNB is driven by a $55 \mathrm{MHz}$ sine-wave with $1.2 \mathrm{~V}_{\mathrm{pp}}$ swing.

The DTC INL is defined similarly to that of a DAC: assuming the total number of bits is $N$, the INL at digital code $k$ is then defined as

$$
I N L(k)=\tau(k)-\frac{k}{2^{N-1}} \cdot \tau_{F S}
$$

where $\tau(k)$ is the measured delay at code $k$ and $\tau_{\mathrm{FS}}$ is the measured full-scale delay.

The simulated INL is shown in Fig. 13 with 200 simulation steps at 100ps full-scale. The maximum INL error is less than $50 \mathrm{fs}(0.05 \%)$ and mainly due to non-ideality in the ramp generation such as the residual current-source nonlinearity. Some uncertainty in results is likely due to simulation accuracy at such small time resolution (note that the pattern is rather regular).

The RMS jitter was also simulated within a bandwidth up to half of the clock rate, resulting in $109 \mathrm{fs}$ and $99 \mathrm{fs}$ at $\mathrm{V}_{\text {st }}$ of $0 \mathrm{mV}$ and $200 \mathrm{mV}$ respectively. Lower jitter at higher $\mathrm{V}_{\text {st }}$ is due to the smaller charging time so less noise integration. The LNB alone is simulated to have a jitter of $81 \mathrm{fs}$, which is the biggest contribution due to its low-slope sine-wave input. 


\section{MEASUREMENTS}

The chip design shown in Fig. 9 was fabricated in 65nm CMOS and a chip photo is shown in Fig. 14. The active area of the DTC-core and DAC is about $0.1 \mathrm{~mm}^{2}$, each taking roughly half. The chip is packaged in a 32-pin Heat-sink Very-thin Quad Flat-pack No-leads (HVQFN) package. All measurements were performed on PCB.

\section{A. Delay INL}

Fig. 15 shows a simplified setup used to measure DTC delay and INL in our time-domain experiment. The chip (DUT) receives a $1.2 \mathrm{Vpp}$ sine-wave input from the $55 \mathrm{MHz}$ crystal oscillator (XO) and delivers a $0.6 \mathrm{Vpp}$ rectangular-wave output to a $50 \mathrm{Ohm}-\mathrm{Z}_{\text {in }}$ oscilloscope (OSP). The crystal signal is also used as reference to trigger the sampling oscilloscope. The chip is programmed from a computer via an integrated two-pin serial-bus interface. Using this setup, we estimated the deterministic part of the INL to be in the order of 150fs at 102ps fullscale delay (0.15\%) and 250fs at 304ps full-scale (0.08\%). However, the results contain large measurement uncertainties on the same order as the estimated INL therefore it is difficult to assess the reliability of these measurements and draw conclusions.

Because the time-domain method is not good enough to directly measure the INL of the chip, we developed an indirect method for the characterization of the DTC-core, that avoids the oscilloscope and instead uses a spectrum analyzer. The basic idea is to periodically modulate the delay of the DTC between two distinct values, which results in a spur [27]. Such a spur can be measured with high fidelity in the frequency domain, as only noise and interference in a small frequency band around the spur frequency will pollute the results. In contrast, a sub-sampling oscilloscope is wideband, and hence sensitive to noise and interference in a wide band.

The proposed measurement setup is shown in Fig. 16. The on-chip DAC is off as its serial digital interface is too slow for the modulation frequency. Instead, an external DAC (Agilent 
M8190A Arbitrary Waveform Generator) was used to produce a square-wave voltage ( $\left.\mathrm{V}_{\text {ext }}\right)$ that switches slowly compared to the input $\mathrm{XO}\left(f_{\mathrm{Vext}}=2.5 \mathrm{MHz}, f_{\mathrm{XO}}=55 \mathrm{MHz}\right)$. We used $10 \mathrm{bits}$ as our full-scale out of the DAC's 14-bit maximum range. We measured the external DAC performance and found that its INL is below +/-0.5LSB (0.05\% referring to 10-bit full-scale) which is not the bottleneck in our DTC-INL measurement.

Note that the start voltage now is defined by $V_{\text {ext }}$, so each voltage level determines a position of the DTC output rising edge; a square wave at $V_{\text {ext }}$ produces a delay/phase modulation at the DTC output, because its rising edge jumps periodically between two positions. This phase modulation appears in the frequency domain as a couple of sidebands, where the strongest occurs at an offset frequency $f_{\mathrm{v}_{\mathrm{ext}}}$ from $f_{X O}$ which is the carrier frequency of the DTC output (see Fig. 16). These sidebands can be measured using a spectrum analyzer. Only the rising edges of DTC output are programmable, therefore a frequency divider by 2 is inserted between the DTC chip and the spectrum analyzer, in order to discard the falling edges of the DTC output.

Just like the modulating signal $\mathrm{V}_{\text {ext }}$, the phase change of the signal at the divider output is also a square wave. By using the standard modulation theory [28], it can be shown that the relative strength of the first sideband (either on the left or right side of the carrier, see Fig. 16) in $\mathrm{dBc}$ is related to the delay step produced by the square wave as the following equation [27]:

$$
\text { spur_dBc }=20 \log _{10}\left(\frac{\tau_{h}}{T_{c k}}\right)
$$

where $\tau_{h}$ is the delay step of the rising edge, produced by the voltage step of the h-th square wave $\mathrm{V}_{\text {ext }}$, and $\mathrm{T}_{\mathrm{ck}}$ is the period of the DTC output.

To achieve high accuracy in spur measurements, it is beneficial to nominally always measure the same spur strength: range switching in a spectrum analyzer is avoided in that way and the nonlinearities in the power detector are minimized. In terms of DAC codes, in one 
code-sweep the full code range is covered with 40 identical code steps. Each code step produces a square wave in terms of $\mathrm{V}_{\text {st }}$, where the amplitude is fixed (so nominally equal delay steps and spur strength), but DC levels are increasing from one code step to the next. For each code step, we measure a spur level which is then converted to a delay step $\tau_{h}$ via (7). Due to nonlinearity there will be variations in the measured $\tau_{h}(1 \leq h \leq 40)$. These variations correspond to DNL errors which can be calculated with the following equation:

$$
\operatorname{DNL}(h)=\tau_{h}-\tau_{i d}
$$

Where $\tau_{i d}$ is the ideal delay step produced by each square wave, which is estimated as the average of all measured $\tau_{h}$ values for a complete code-sweep. The INL is the cumulative sum of the DNL.

Both low-frequency and high-frequency noise exist in the measurements. We chose to do each sweep (40 points) within 10 minutes, and then repeat the procedure 50 times, so 50 nominally equal data sets result. In this way, a single INL plot of each sweep is less sensitive to low-frequency noise, and an average of 50 helps to remove high-frequency noise.

The INL curve from this method for a full-scale delay of 71ps, using 40 delay-steps, is shown in Fig. 17. Both the left and right spur-sidebands were measured and they agree within about 50fsec with each other.

The two y-axes in Fig. 17 indicate, respectively, the absolute INL in fs, and its normalized value to the full-scale delay, i.e. INL in percentage. The absolute INL is within 235fs. The normalized INL is within $0.33 \%$, corresponding to an effective resolution of $\log _{2}(1 \div$ $0.33 \%)=8.2$ bits, when only considering the INL-limitation.

The measured INL is the combination of the chip and the external DAC, while the on-chip DAC is not involved. The external DAC is a voltage-mode DAC instead of a current-DAC, therefore the linear $R_{D A C}$ in Fig. 10 is not effective in this case to help the nonlinear $R_{\text {on }}$ of $\mathrm{M}_{\mathrm{N} 1}$. As shown in Fig. 18, $\mathrm{V}_{\text {ext }}$ goes through an extra on-chip static switch $\mathrm{M}_{\mathrm{sw}}$, which 
contributes additional nonlinearity. Experiments show that using external voltage-DAC degrades INL and limits the linear $\mathrm{V}_{\text {ext }}$ range to about $100 \mathrm{mV}$, reducing the linear full-scale range, compared to the case with an on-chip current-DAC.

The full-scale delay is varied roughly from 20-to-200ps. The measured INL of 19ps and 189ps full-scales are shown in Fig. 19 and Fig. 20, respectively. The maximum INL is 64fs for 19ps full-scale (normalized INL=0.34\%, 8.2bits), and 328fs for 189ps full-scale (normalized INL=0.17\%, 9.2bits), showing good linearity over a large delay range.

Very different settings are used for these different full-scales, in terms of DAC voltage range, charging current value, and charging capacitor value. The different contributions to nonlinearity (such as current source, switch resistance, parasitic capacitance, and DAC) will increase or decrease at different settings. Therefore, different subtle nonlinearity mechanism can be dominant at different full-scales, so the INL shape or even polarity can change. It is difficult to exactly pinpoint all mechanisms and match them to a model. On the other hand, we repeated many of the measurements and find reproducible results, while the measured curves from the two spur-sidebands also match each other in all three cases. These results indicate that very competitive performance can be achieved.

\section{B. Phase Noise and Jitter}

As mentioned in section III.E, the simulated jitter is about 100fs, which is less than the jitter of the oscilloscope we used. Hence a time-domain measurement was meaningless. Since the DTC is running at the crystal frequency, direct phase-noise measurement is also challenging, as it represents a very low phase-noise level at $55 \mathrm{MHz}$ carrier. Also the measurement should only be sensitive to the rising edge of the DTC output.

In an attempt to still quantify the phase noise, we used a previously published low-jitter PLL [29] as a frequency multiplier with the setup in Fig. 21 (a). Within the PLL loop bandwidth, the DTC noise is conveyed to its VCO output. As the VCO runs at a much higher 
frequency than the reference clock (2.2GHz versus $55 \mathrm{MHz})$, a given timing jitter corresponds to more phase variation making phase-noise measurements easier. The on-chip DAC is used in the noise measurement.

At the DTC setting with about 100ps full-scale delay, the total measured phase noise from the DTC and the PLL together is shown in Fig. 21 (b). The in-band phase noise floor at $2 \mathrm{MHz}$ is $-124 \mathrm{dBc} / \mathrm{Hz}$ at a $2.2 \mathrm{GHz}$ carrier with less than $\pm 1 \mathrm{~dB}$ variation for all digital codes, while the PLL alone without DTC showed $-125 \mathrm{dBc} / \mathrm{Hz}$ [29]. This shows the DTC is suitable for low-phase-noise applications. The integrated jitter from $100 \mathrm{kHz}$ to $100 \mathrm{MHz}$ is $210 \mathrm{fs}$ for the DTC and the PLL together, at a loop bandwidth of 5MHz. Note that the DTC should only contribute significantly to the noise within the loop bandwidth due to the PLL low-pass transfer function from the reference path to the VCO output.

\section{Benchmark}

Table 1 compares this DTC with other recent work. This work demonstrates the finest time resolution and achieves the best INL when benchmarked at a similar full-scale delay. To evaluate a DTC design, it is more appropriate to compare INL for similar full-scale delays, because not only absolute INL but also normalized INL often changes with full-scale delay for the same DTC. At similar full-scale and in terms of INL in percentage, at 71ps delay compared to [5] and [7] the INL is 15x better; at 189ps delay compared to [1] and [2] the INL is $6 \mathrm{x}$ better. A recent DTC [4] shows a similar INL in percentage $(0.18 \%)$, but our work achieves this INL at a 3x smaller full-scale delay and 3x finer resolution. Note that achieving the same INL in percentage at a smaller full-scale delay is more difficult, as small absolute delay errors become more relevant. Based on information provided in section IV.A, we actually expect that using on-chip DAC would give even better INL.

This work is also competitive in terms of jitter, and certainly for in-band phase noise when applied in a PLL. At 1.2VDD, 55MHz input, and 102ps delay, the power consumption of the 
DTC-core is $0.8 \mathrm{~mW}\left(\mathrm{I}_{\mathrm{b}}=260 \mathrm{uA}\right)$ which can be lowered with process scaling, and the DAC consumes $1 \mathrm{~mW}$ which can be lowered by a customized design for $55 \mathrm{MHz}$ speed.

\section{CONCLUSIONS}

This paper has shown that the popular variable-slope delay method suffers from INL due to the variable slope of the input ramp voltage in combination with bandwidth limitations and the transition through different operating modes of the threshold comparator. A constant-slope method is proposed that generates delay by varying the start voltage of a ramp instead of its slope, which strongly improves INL.

A DTC chip based on this method is implemented in a 65nm CMOS. It receives a sine wave as input and delivers a digitally-controlled time-delayed clock edge at the output. A 10-bit DAC defines the start voltage of the critical constant-slope ramp.

The DTC INL was measured using a newly developed frequency-domain method, detecting a spur generated by modulating the DTC phase. Measurement results show that the INL is within 328fs for 189ps full-scale delay (0.17\%) and within 64fs for 19ps full-scale delay $(0.34 \%)$

\section{ACKNOWLEDGMENTS}

We acknowledge the Dutch government for project funding in the frame of "the SPITS Kennerswerkersregeling project" and NXP Semiconductors for sponsoring chip fabrication. We sincerely thank G. van der Weide, N. Pavlovic, H. Brekelmans, X. He, J. van Sinderen, D. Schinkel, and R. Roovers for discussions and tape-out assistance. We also sincerely thank G. Wienk, H. de Vries, J. Velner, T. Aarnink, X. Gao, and M. Soer for help during the multi-year period of measurements and many discussions that finally lead to the newly developed spurdetection based measurement method. 


\section{REFERENCES}

[1] Pavlovic, N.; Bergervoet, J.; , "A 5.3GHz digital-to-time-converter-based fractional-N alldigital PLL," IEEE International Solid-State Circuits Conference (ISSCC), pp.54-56, 2024 Feb. 2011

[2] Tasca, D.; Zanuso, M.; Marzin, G.; Levantino, S.; Samori, C.; Lacaita, A.L.; , "A 2.9-to4.0GHz fractional-N digital PLL with bang-bang phase detector and 560fsrms integrated jitter at 4.5mW power," IEEE International Solid-State Circuits Conference (ISSCC), pp.88-90, 20-24 Feb. 2011

[3] Staszewski, R.B.; Waheed, K.; Vemulapalli, S.; Dulger, F.; Wallberg, J.; Chih-Ming Hung; Eliezer, O.; , "Spur-free all-digital PLL in 65nm for mobile phones," IEEE International Solid-State Circuits Conference (ISSCC), pp.52-54, 20-24 Feb. 2011

[4] N. Markulic, K. Raczkowski, P. Wambacq, J. Craninckx, “A 10-Bit, 550-fs Step Digitalto-Time Converter in 28nm CMOS”, European Solid-State Circuits Conference (ESSCIRC), September 2014

[5] K. Inagaki, D. Antono, M. Takamiya, S. Kumashiro, T. Sakurai, “A 1-ps resolution onchip sampling oscilloscope with 64:1 tunable sampling range based on ramp waveform division scheme” IEEE VLSI Symposium 2006

[6] M. Safi-Harb, G. W. Roberts, “70-GHz Effective Sampling Time-Base On-Chip Oscilloscope in CMOS”, IEEE JSSC, 2007 August

[7] T. Okayasu, M. Suda, K. Yamamoto, S. Kantake, S. Sudou, D. Watanabe, “1.83psResolution CMOS Dynamic Arbitrary Timing Generator for >4GHz ATE Applications”, IEEE ISSCC 2006 
[8] S. Talwalkar, T. Gradishar, B. Stengel, G. Cafaro, G. Nagaraj, “Controlled Dither in 90nm Digital To Time Conversion Based Direct Digital Synthesizer for Spur Mitigation”, IEEE RFIC Symposium 2010

[9] Yong-Chang Choi, Sang-Sun Yoo, and Hyung-Joun Yoo, "A fully digital polar transmitter using a digital-to-time converter for high data rate system," IEEE International Symposium on Radio-Frequency Integration Technology, RFIT, pp. 56-59, 2009.

[10] D. Zito, D. Pepe, M. Mincica, and F. Zito, "A 90nm CMOS SoC UWB pulse radar for respiratory rate monitoring," IEEE International Solid-State Circuits Conference (ISSCC), pp. 40-41, 2011.

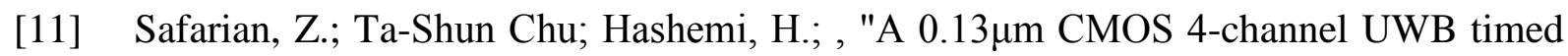
array transmitter chipset with sub-200ps switches and all-digital timing circuitry," IEEE Radio Frequency Integrated Circuits Symposium, RFIC, pp.601-604, June 2008

[12] Poulton, K.; Neff, R.; Muto, A.; Liu, W.; Burstein, A.; Heshami, M., "A 4GSample/s 8b ADC in 0.35um CMOS," IEEE International Solid-State Circuits Conference (ISSCC), Feb. 2002

[13] Klumperink, E.A.M.; Klein, Carlo T.; Ruggeberg, B.; van Tuijl, E.J.M., "AM suppression with low AM-PM conversion with the aid of a variable-gain amplifier," SolidState Circuits, IEEE Journal of , vol.31, no.5, pp.625,633, May 1996

[14] J. Buckwalter and A. Hajimiri, "An active analog delay and the delay reference loop," IEEE RFIC Symposium, 2004

[15] S. K. Garakoui, E. A. M. Klumperink, B. Nauta and F. E. van Vliet, "A 1-to-2.5GHz phased-array IC based on gm-RC all-pass time-delay cells," IEEE ISSCC, 2012

[16] Levantino, S.; Marzin, G.; Samori, C., "An Adaptive Pre-Distortion Technique to Mitigate the DTC Nonlinearity in Digital PLLs," IEEE Journal of Solid-State Circuits,vol.49, no.8, pp.1762,1772, Aug. 2014 
[17] S. Alahdab, A. Mantyniemi, J. Kostamovaara, “A 12-Bit Digital-to-Time Converter (DTC) with sub-ps-level resolution using current DAC and differential switch for Timeto-Digital Converter (TDC)”, International Instrumentation and Measurement Technology Conference (I2MTC), 2012

[18] Lee, T.H.; Donnelly, K.S.; Ho, J.T.C.; Zerbe, J.; Johnson, M.G.; Ishikawa, T., "A 2.5 V CMOS delay-locked loop for 18 Mbit, 500 megabyte/s DRAM," IEEE Journal of SolidState Circuits, vol.29, no.12, pp.1491,1496, Dec 1994

[19] S. Sidiropoulos and M. Horowitz, “A semidigital dual delay-locked loop”, IEEE Journal of Solid-State Circuits, November 1997.

[20] J. van Valburg and R. J. van de Plassche, “An 8-b 650-MHz folding ADC,” IEEE J. Solid-State Circuits, vol. 27, no. 12, pp. 1662-1666, Dec. 1992.

[21] Henzler, S.; Koeppe, S.; Lorenz, D.; Kamp, W.; Kuenemund, R.; Schmitt-Landsiedel, D., "A Local Passive Time Interpolation Concept for Variation-Tolerant High-Resolution Time-to-Digital Conversion," IEEE Journal of Solid-State Circuits, July 2008

[22] Zanuso, M.; Levantino, S.; Samori, C.; Lacaita, A.L.; , "A Wideband 3.6 GHz Digital $\Delta \Sigma$ Fractional-N PLL With Phase Interpolation Divider and Digital Spur Cancellation," IEEE Journal of Solid-State Circuits, vol.46, no.3, pp.627-638, March 2011

[23] R. van de Plassche, "CMOS Integrated Analog-to-Digital and Digital-to-Analog Converters”, $2^{\text {nd }}$ Edition, Kluwer Academic Publishers, 2003

[24] M. Rosario, P. Gaetano, P. Massimo, "Propagation Delay of an RC-Chain with a Ramp Input”, IEEE Trans. on Circuits and Systems - II: Express Briefs, Jan. 2007

[25] J. M. Daga, D. Auvergne, “A Comprehensive Delay Macro Modeling for Submicrometer CMOS Logics”, IEEE Journal of Solid-State Circuits, Jan. 1999 
[26] X. Gao; E. Klumperink; G. Socci; M. Bohsali; B. Nauta; , "A 2.2GHz sub-sampling PLL with $0.16 \mathrm{psrms}$ jitter and $-125 \mathrm{dBc} / \mathrm{Hz}$ in-band phase noise at $700 \mu \mathrm{W}$ loopcomponents power," IEEE Symposium on VLSI Circuits, June 2010

[27] C. Palattella, E. Klumperink, Z. Ru, B. Nauta, “A sensitive method to measure the integral nonlinearity of a digital-to-time converter, based on phase modulation”, accepted by IEEE TCAS-II

[28] S. Haykin, Communication Systems, 4th ed. Wiley, 2000

[29] X. Gao; E. Klumperink; M. Bohsali; B. Nauta; , "A 2.2GHz 7.6mW sub-sampling PLL with $-126 \mathrm{dBc} / \mathrm{Hz}$ in-band phase noise and 0.15 psrms jitter in $0.18 \mu \mathrm{m}$ CMOS," IEEE International Solid-State Circuits Conference (ISSCC), Feb. 2009

\section{List of Figures and Tables}

Fig. $1 \mathrm{~A}$ voltage ramp generated by a current source charging a capacitor, and delay $\mathrm{t}_{\mathrm{d}}$ defined by the ramp crossing $\mathrm{V}_{\mathrm{d}}$

Fig. 2 Principle of (a) variable-slope method in which different slopes at comparator input define delay; (b) constant-slope method in which different start-voltages at comparator input define delay.

Fig. 3 Phase interpolation concept starting from two equal-slope signals $V_{A}$ and $V_{B}$, where the interpolated phases $\left(V_{1}-V_{3}\right)$ have the same slope for the part within the two dashed lines

Fig. 4 Illustration of constant-slope method using practical ramps which ideally would start with a constant-slope part at least to $\mathrm{V}_{\text {st,max }}$ then share a constant-shape part beyond $\mathrm{V}_{\text {st,max }}$

Fig. 5 Delay mechanism for constant-slope method: the start voltage $\mathrm{V}_{\text {st }}$ linearly affects delay $\tau$, while the comparator response is identical for ramp $a$ and $b$ because it "sees" the same shape of both ramps 
Fig. 6 INL simulation bench to compare the variable and constant slope methods

Fig. 7 Simulated INL for the variable-slope method with 110ps full-scale delay: 1.4ps maximum INL results

Fig. 8 Simulated INL for the constant-slope method at 100ps full-scale delay: only $15 \mathrm{fs}$ maximum INL is found compared to 1.4ps in Fig. 7

Fig. 9 Block diagram of the implemented DTC (LNB = Low-Noise Buffer)

Fig. 10 Circuit schematic of the DTC-core: B1/B2/B3 are buffers made of two inverters all using regular- $\mathrm{V}_{\text {th }}$ MOSFET; D1/D2 are drivers made of two inverters using a mix of regular$\mathrm{V}_{\text {th }}$ and high- $\mathrm{V}_{\text {th }}$ MOSFET as shown in Fig. 11

Fig. 11 PMOS driver D1 consisting of two inverters using a mix of regular- $\mathrm{V}_{\text {th }}$ and high- $\mathrm{V}_{\text {th }}$ MOSFET to produce 1/3 duty cycle; input and output waveforms of the low-noise buffer showing its PMOS is only on for $\sim 1 / 3$ duty cycle when the lower two NMOS FETs are off

Fig. 12 Pulse generator producing $\mathrm{V}_{\text {res }}$ used in Fig. 10 with nominal 0.7ns width; the 20fF contributes to $\tau_{1}$ which helps $\mathrm{V}_{\text {res }}$ to fit in the overall timing plan while the $100 \mathrm{fF}$ contributes to $\tau_{2}$ which determines the $V_{\text {res }}$ pulse width

Fig. 13 Simulated INL of the DTC-core at 100ps full scale, with ideal DAC, using a PSP model for the 65nm CMOS transistors

Fig. 14 Chip photo of the DTC realized in 65nm CMOS with active area of $0.1 \mathrm{~mm}^{2}$

Fig. 15 Setup for INL measurements in the time domain using an oscilloscope

Fig. 16 Setup for INL measurements in the frequency domain using the method of [27]

Fig. 17 Measured INL at 71ps full-scale using the proposed method $\left(\mathrm{V}_{\mathrm{ext}}=0\right.$ to $\left.64.8 \mathrm{mV}\right)$; the $\mathrm{L}$ and $\mathrm{R}$ curves refer to measurements from the left and right spur-sidebands respectively 
Fig. 18 Using an external voltage-mode DAC where the voltage signal $\mathrm{V}_{\text {ext }}$ goes through an extra static switch $\mathrm{M}_{\mathrm{sw}}$ which contributes additional INL, and the linear $\mathrm{R}_{\mathrm{DAC}}$ is not effective to reduce the nonlinearity of $\mathrm{M}_{\mathrm{N} 1}$ compared to using current-DAC

Fig. 19 Measured INL at 19ps full-scale using the proposed method $\left(\mathrm{V}_{\mathrm{ext}}=0\right.$ to $\left.33.6 \mathrm{mV}\right)$

Fig. 20 Measured INL at 189ps full-scale using the proposed method ( $\mathrm{V}_{\mathrm{ext}}=0$ to $\left.101.5 \mathrm{mV}\right)$

Fig. 21 (a) Setup for phase noise measurement; (b) Measured phase noise of the DTC as a reference buffer for a low-jitter PLL (reference spur at 55MHz)

Table 1 Comparison with other recent work on DTC performance

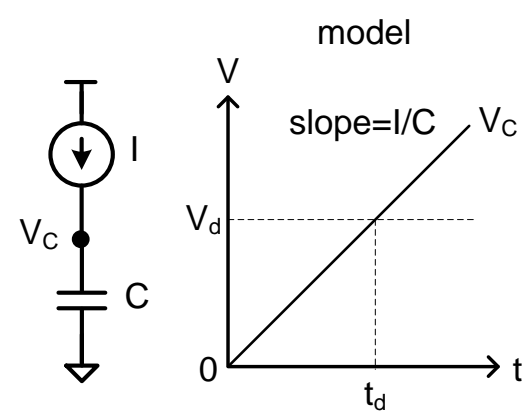

(a)

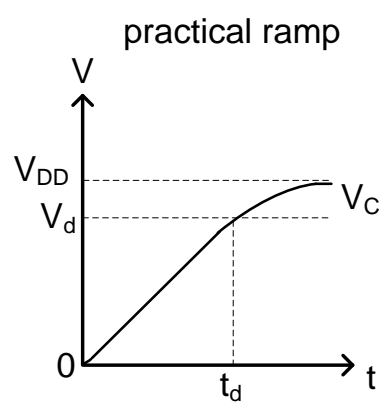

(b)

Fig. 1 A voltage ramp generated by a current source charging a capacitor, and delay $t_{d}$ defined by the ramp crossing $\mathrm{V}_{\mathrm{d}}$ 
Ramp

Threshold

Generation

Comparison

(a)
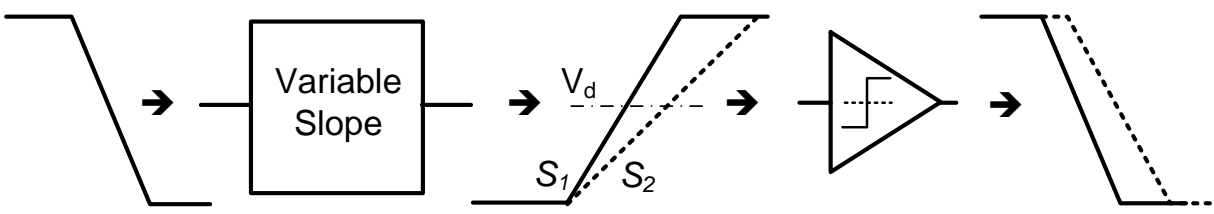

(b)

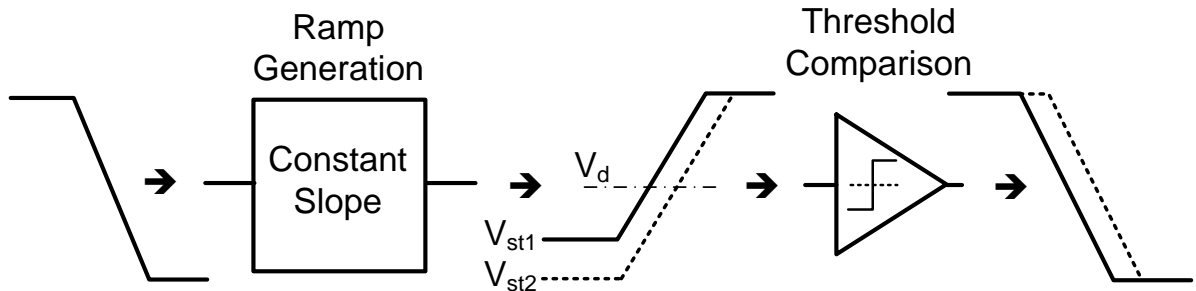

Fig. 2 Principle of (a) variable-slope method in which different slopes at comparator input define delay; (b) constant-slope method in which different start-voltages at comparator input define delay.

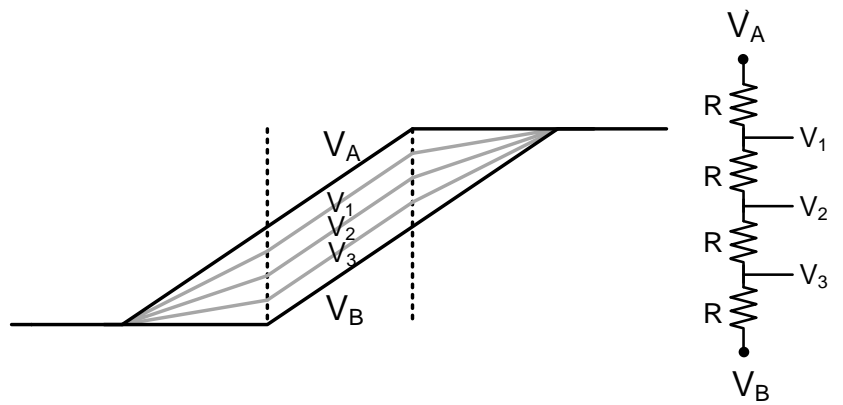

Fig. 3 Phase interpolation concept starting from two equal-slope signals $V_{A}$ and $V_{B}$, where the interpolated phases $\left(V_{1}-V_{3}\right)$ have the same slope for the part within the two dashed lines

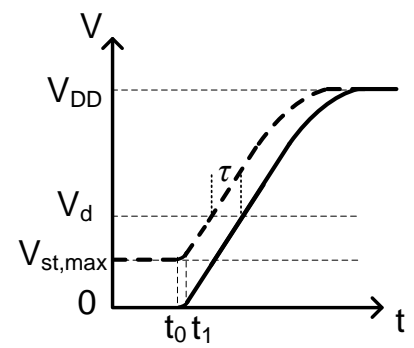

Fig. 4 Illustration of constant-slope method using practical ramps which ideally would start with a constant-slope part at least to $\mathrm{V}_{\text {st,max }}$ then share a constant-shape part beyond $\mathrm{V}_{\text {st,max }}$ 


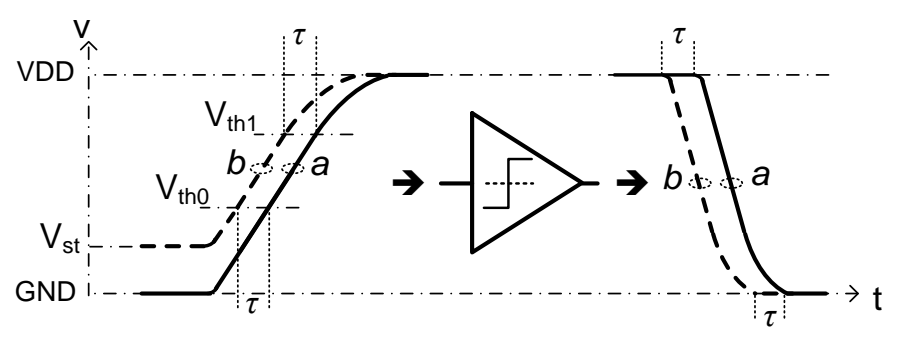

Fig. 5 Delay mechanism for constant-slope method: the start voltage $\mathrm{V}_{\text {st }}$ linearly affects delay $\tau$, while the comparator response is identical for ramp $a$ and $b$ because it "sees" the same shape of both ramps

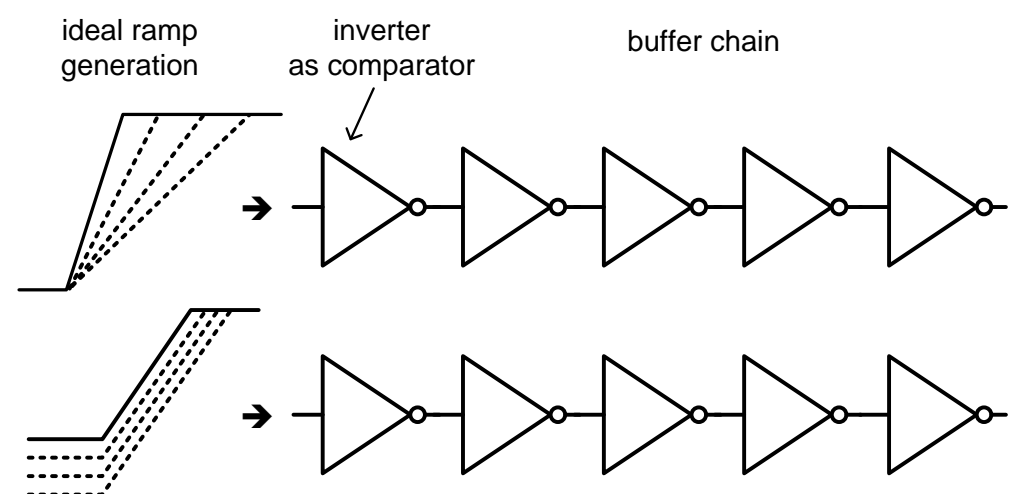

Fig. 6 INL simulation bench to compare the variable and constant slope methods

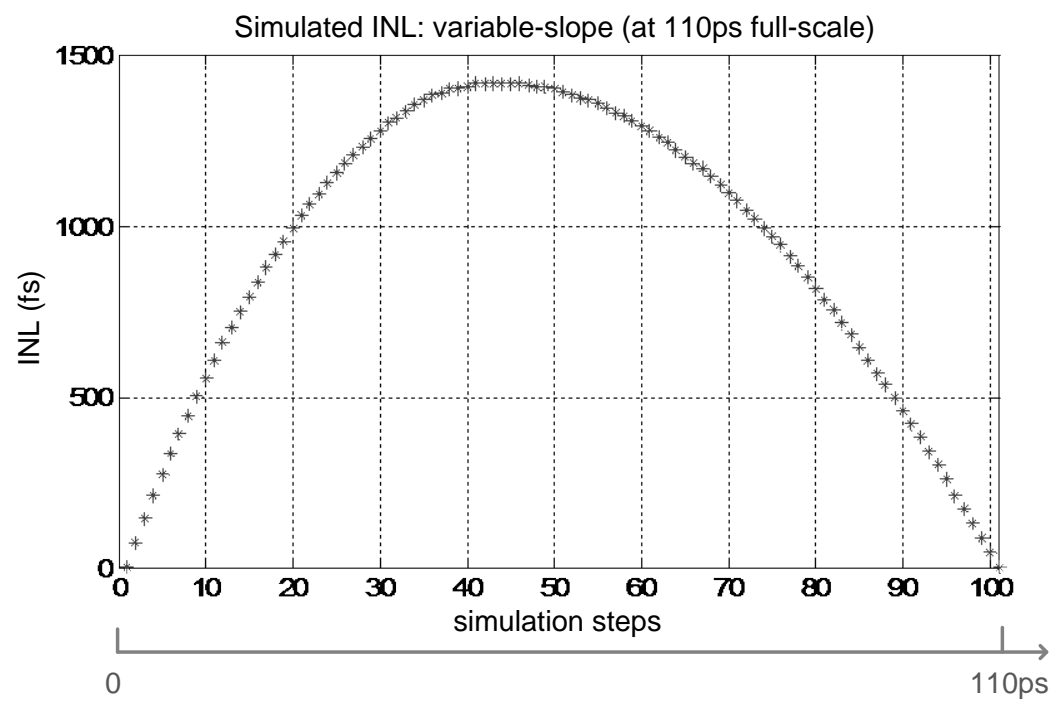

Fig. 7 Simulated INL for the variable-slope method with 110ps full-scale delay: 1.4ps maximum INL results 


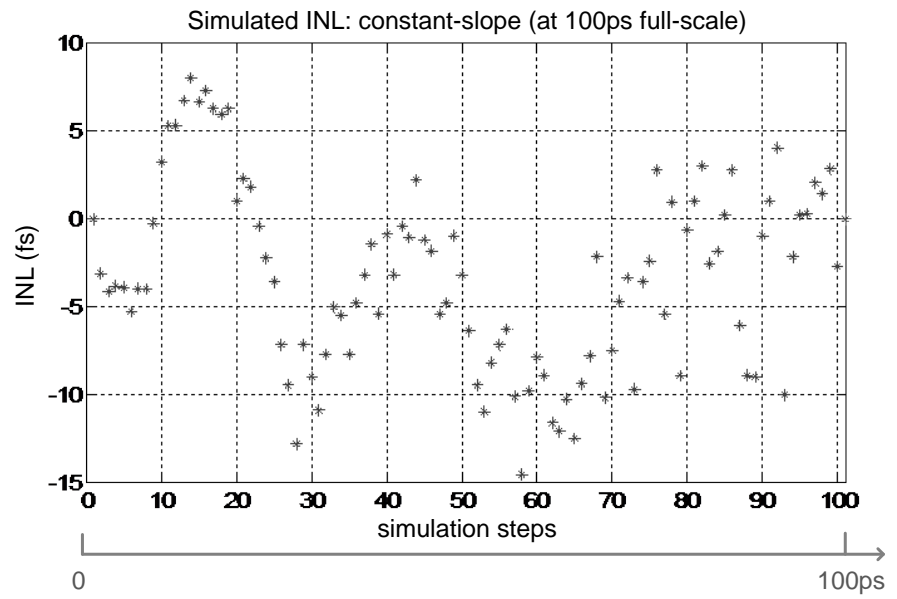

Fig. 8 Simulated INL for the constant-slope method at 100ps full-scale delay: only $15 \mathrm{fs}$ maximum INL is found compared to 1.4 ps in Fig. 7

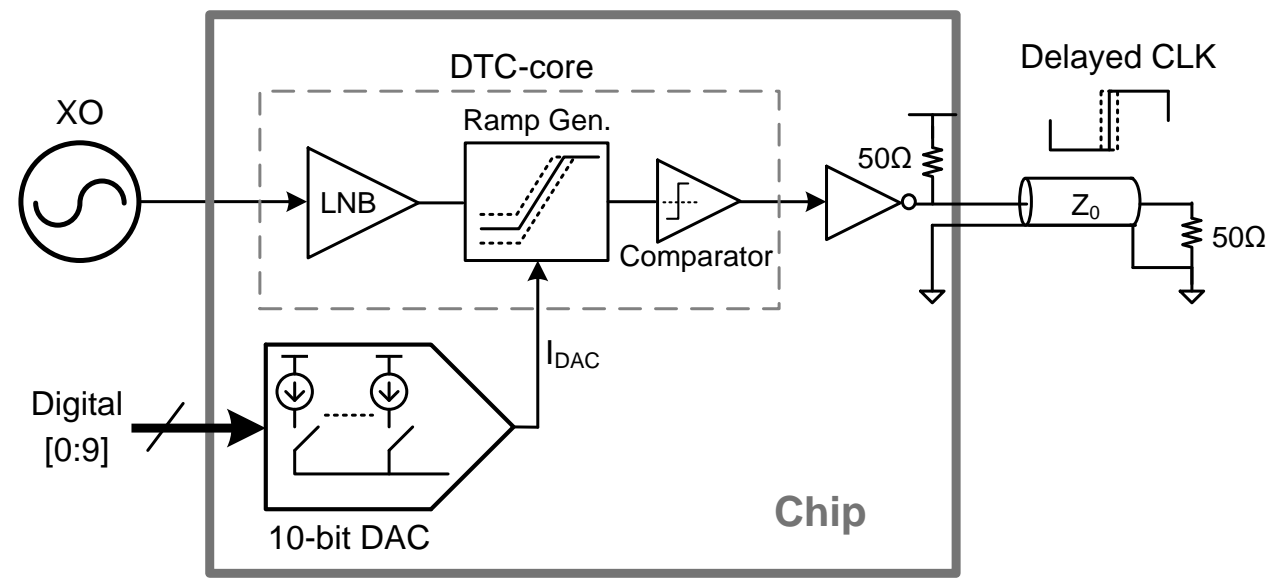

Fig. 9 Block diagram of the implemented DTC (LNB = Low-Noise Buffer) 


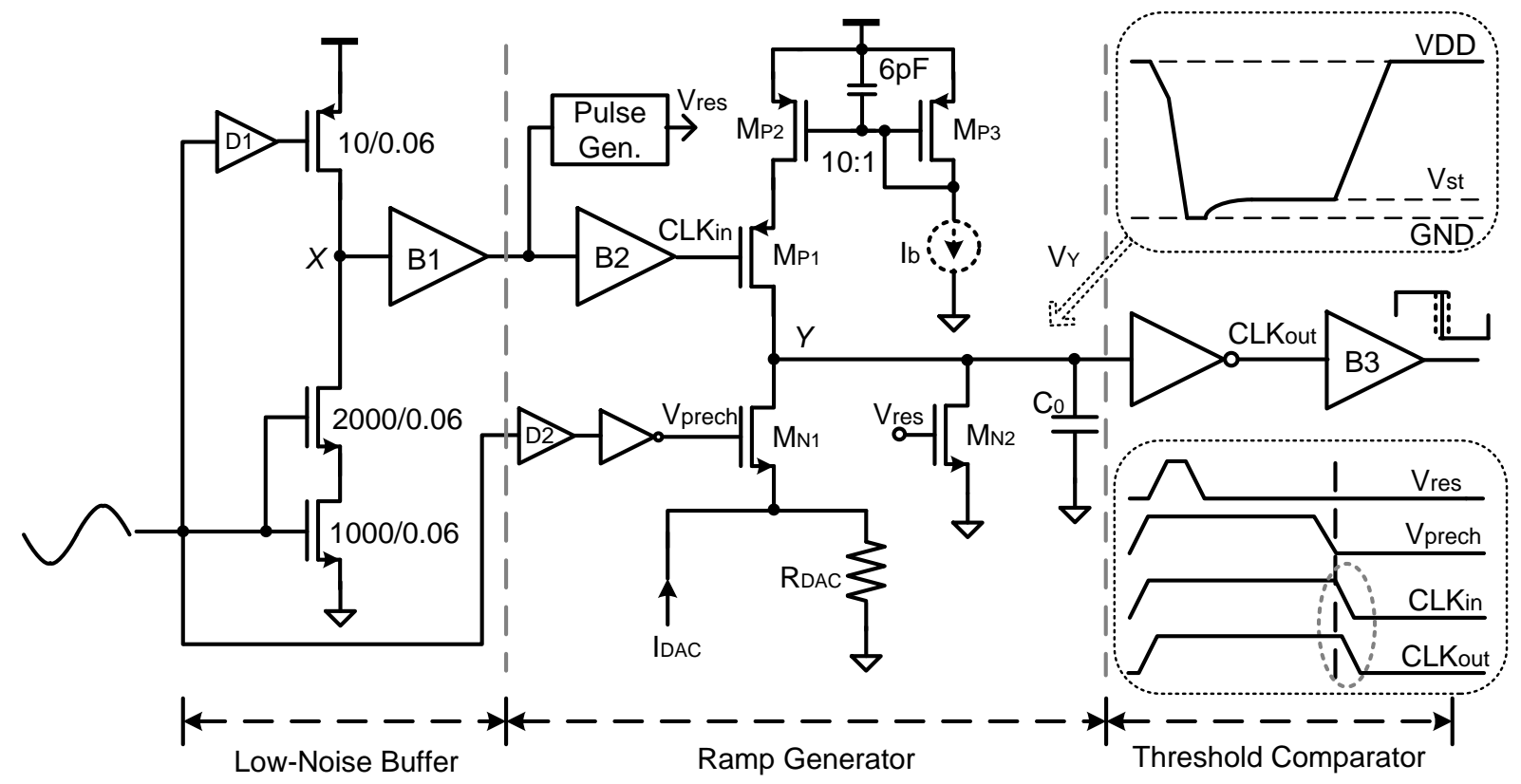

Fig. 10 Circuit schematic of the DTC-core: B1/B2/B3 are buffers made of two inverters all using regular- $\mathrm{V}_{\text {th }}$ MOSFET; D1/D2 are drivers made of two inverters using a mix of regular$\mathrm{V}_{\text {th }}$ and high- $\mathrm{V}_{\text {th }}$ MOSFET as shown in Fig. 11

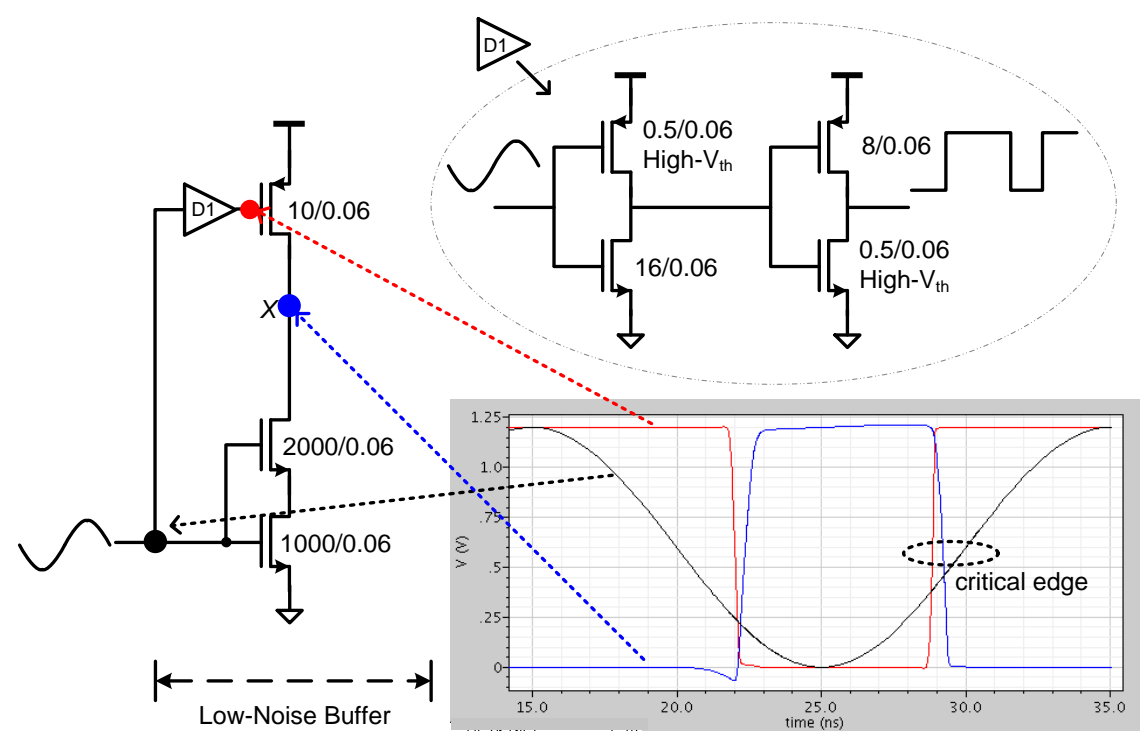

Fig. 11 PMOS driver D1 consisting of two inverters using a mix of regular- $\mathrm{V}_{\text {th }}$ and high- $\mathrm{V}_{\text {th }}$ MOSFET to produce $~ 1 / 3$ duty cycle; input and output waveforms of the low-noise buffer showing its PMOS is only on for $\sim 1 / 3$ duty cycle when the lower two NMOS FETs are off. 


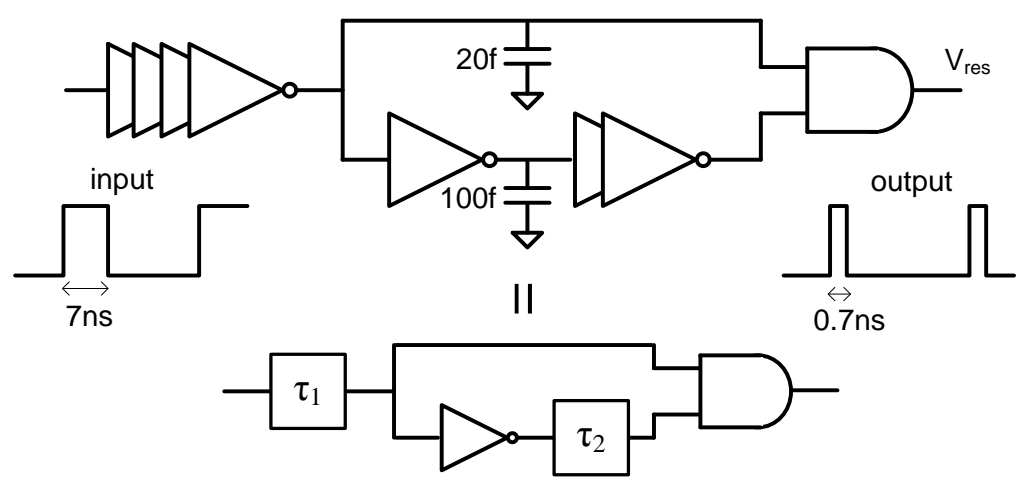

Fig. 12 Pulse generator producing $\mathrm{V}_{\text {res }}$ used in Fig. 10 with nominal 0.7ns width; the 20fF contributes to $\tau_{1}$ which helps $V_{\text {res }}$ to fit in the overall timing plan while the 100fF contributes to $\tau_{2}$ which determines the $V_{\text {res }}$ pulse width

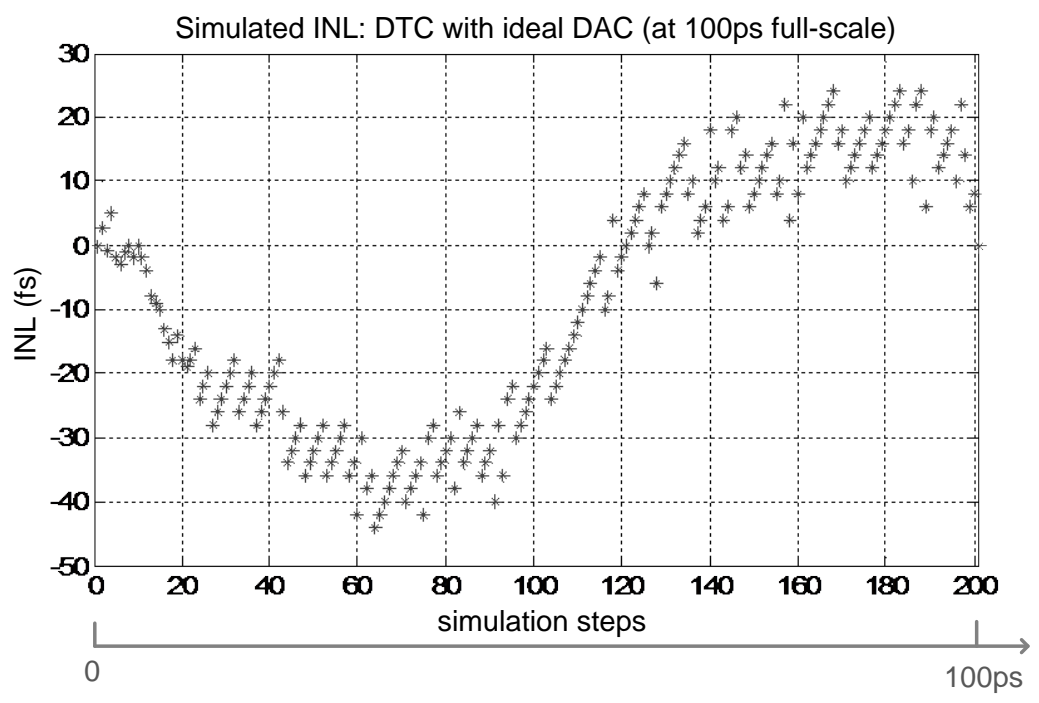

Fig. 13 Simulated INL of the DTC-core at 100ps full scale, with ideal DAC, using a PSP model for the 65nm CMOS transistors

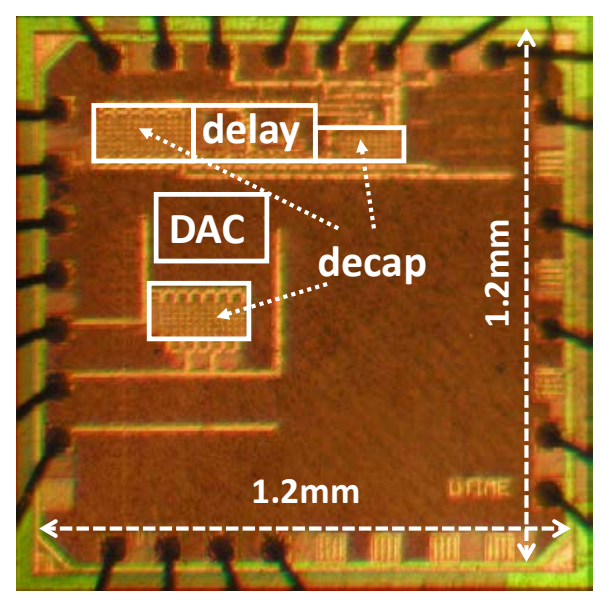

Fig. 14 Chip photo of the DTC realized in 65nm CMOS with active area of $0.1 \mathrm{~mm}^{2}$ 


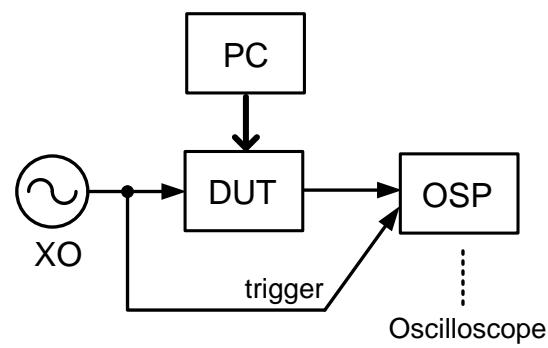

Fig. 15 Setup for INL measurements in the time domain using an oscilloscope
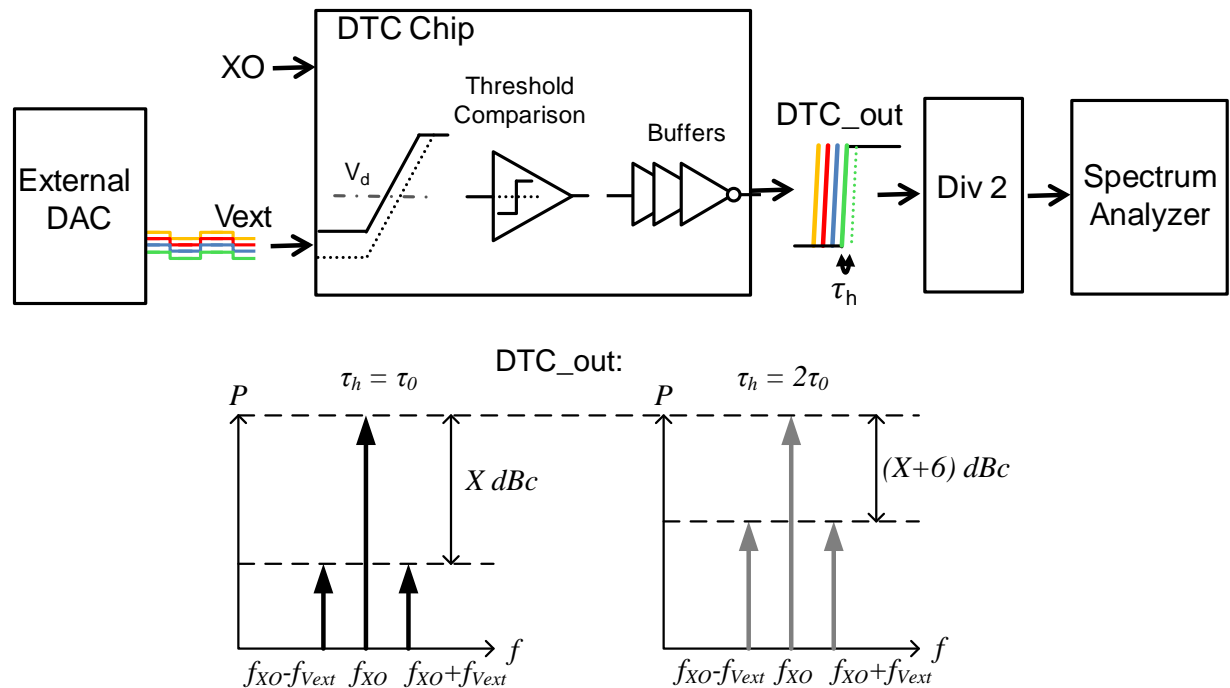

Fig. 16 Setup for INL measurements in the frequency domain using the method of [27].

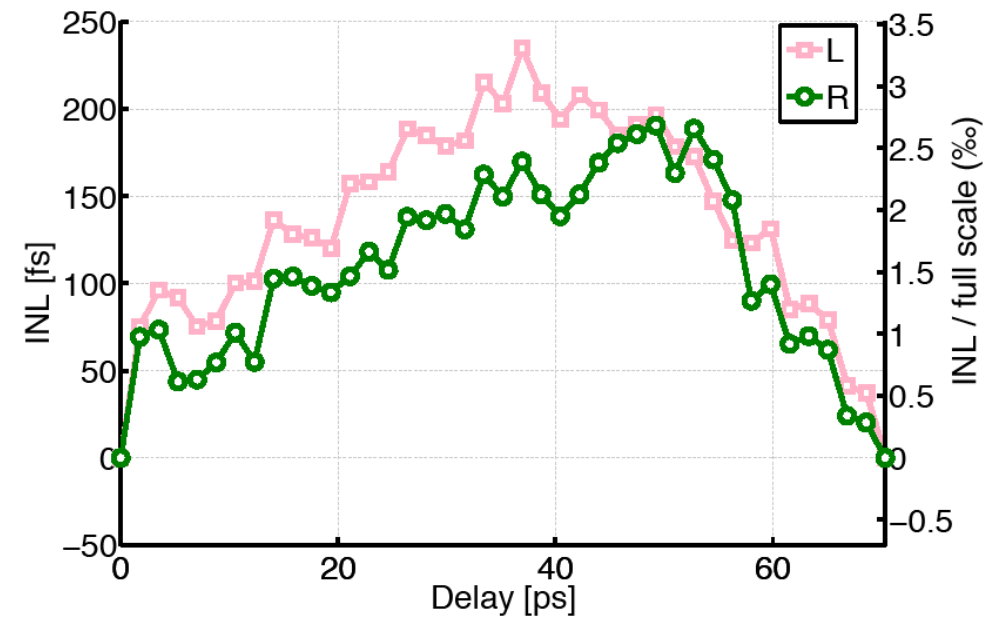

Fig. 17 Measured INL at 71ps full-scale using the proposed method $\left(\mathrm{V}_{\mathrm{ext}}=0\right.$ to $\left.64.8 \mathrm{mV}\right)$; the $\mathrm{L}$ and $\mathrm{R}$ curves refer to measurements from the left and right spur-sidebands respectively. 


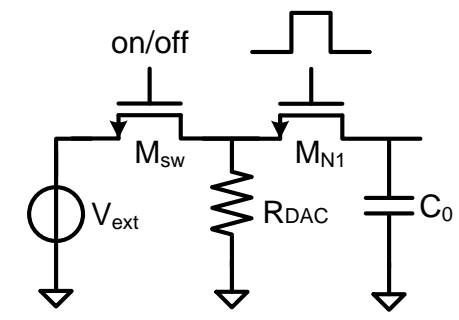

Fig. 18 Using an external voltage-mode DAC where the voltage signal $\mathrm{V}_{\text {ext }}$ goes through an extra static switch $\mathrm{M}_{\mathrm{sw}}$ which contributes additional INL, and the linear $\mathrm{R}_{\mathrm{DAC}}$ is not effective to reduce the nonlinearity of $\mathrm{M}_{\mathrm{N} 1}$ compared to using current-DAC

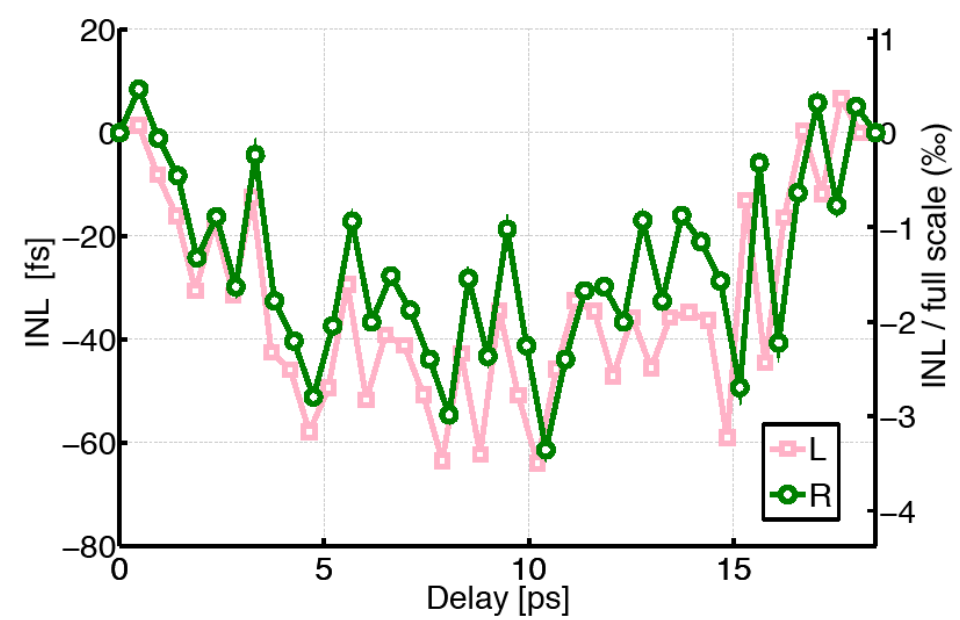

Fig. 19 Measured INL at 19ps full-scale using the proposed method $\left(\mathrm{V}_{\text {ext }}=0\right.$ to $\left.33.6 \mathrm{mV}\right)$

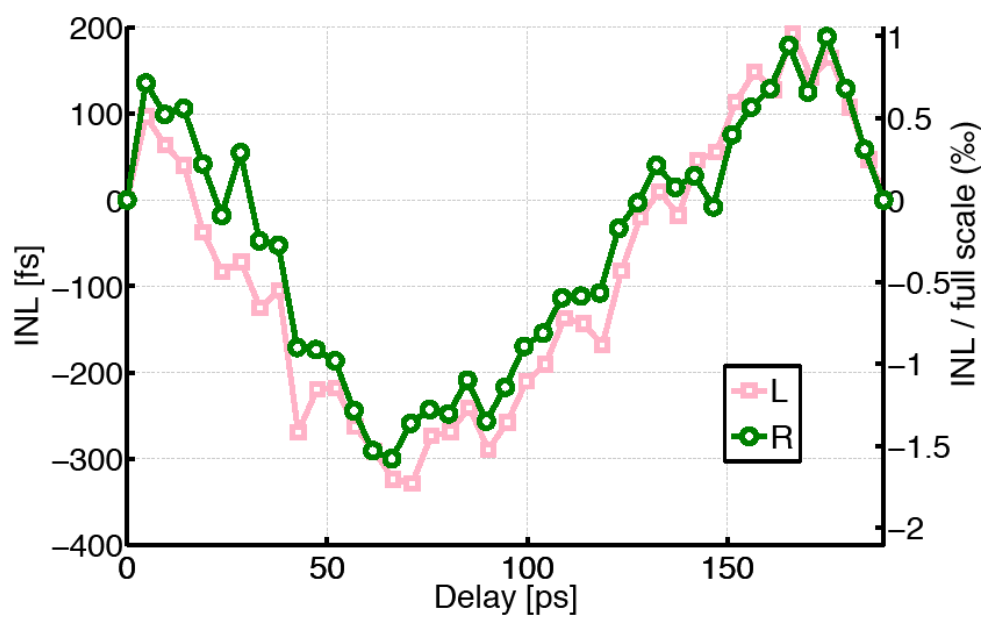

Fig. 20 Measured INL at 189ps full-scale using the proposed method ( $\mathrm{V}_{\text {ext }}=0$ to $101.5 \mathrm{mV}$ ) 
(a)

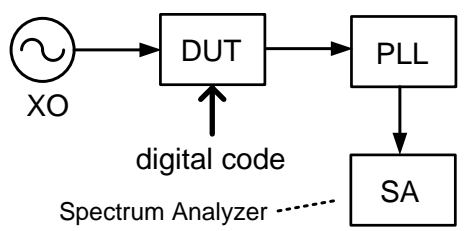

(b)

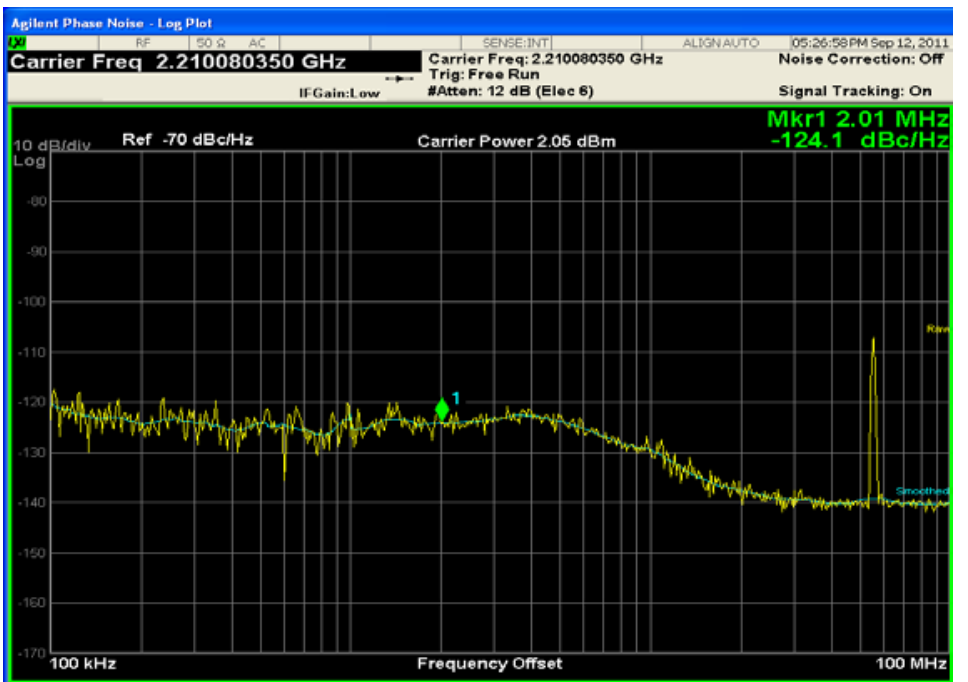

Fig. 21 (a) Setup for phase noise measurement; (b) Measured phase noise of the DTC as a reference buffer for a low-jitter PLL (reference spur at 55MHz)

\begin{tabular}{|c|c|c|c|c|c|c|}
\hline & This Work & $\begin{array}{c}{[1]} \\
\text { ISSCC11 }\end{array}$ & $\begin{array}{c}{[2]} \\
\text { ISSCC11 }\end{array}$ & $\begin{array}{c}{[4]} \\
\text { ESSCIRC14 }\end{array}$ & $\begin{array}{c}{[5]} \\
\text { VLSI06 }\end{array}$ & $\begin{array}{c}{[7]} \\
\text { ISSCC06 }\end{array}$ \\
\hline $\begin{array}{l}\text { Delay } \\
\text { Method }\end{array}$ & $\begin{array}{l}\text { Variable Start-Voltage } \\
\text { (Constant Slope) }\end{array}$ & Variable Slope & Variable Slope & Variable Slope & $\begin{array}{l}\text { Variable } \\
\text { Threshold }\end{array}$ & $\begin{array}{c}\text { Variable } \\
\text { Slope }\end{array}$ \\
\hline $\begin{array}{l}\text { Fine-Delay } \\
\text { Range }\end{array}$ & 19 189ps & $186 \mathrm{ps}$ & 247 338ps & $563 p s$ & $64 \mathrm{ps}$ & $59 p s$ \\
\hline Resolution & 19 185fs & $4700 f s$ & 241 330fs & $550 f s$ & $1000 f s$ & $1830 f s$ \\
\hline INL & 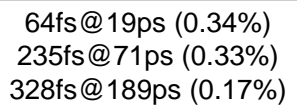 & $\begin{array}{c}1900 f s^{3} @ 186 p s \\
(1 \%)\end{array}$ & $\begin{array}{c}3000 f^{3} @ 305 p s \\
(1 \%)\end{array}$ & $\begin{array}{c}\text { 990fs@563ps } \\
(0.18 \%)\end{array}$ & $\begin{array}{l}\text { 3200fs @ } \\
64 p s(5 \%)\end{array}$ & $\begin{array}{l}\text { 3000fs @ } \\
59 p s(5 \%)\end{array}$ \\
\hline $\begin{array}{l}\text { In-Band PN } \\
(\mathrm{dBc} / \mathrm{Hz})\end{array}$ & $\begin{array}{c}<-124 @ 2.21 \mathrm{GHz}^{1} \\
(-131 @ 1 \mathrm{GHz})\end{array}$ & $\begin{array}{c}<-100 @ 5.38 \mathrm{GHz} \\
(-115 @ 1 \mathrm{GHz})\end{array}$ & $\begin{array}{c}<-102 @ 3.28 \mathrm{GHz} \\
(-112 @ 1 \mathrm{GHz})\end{array}$ & $\begin{array}{l}-155 @ 40 \mathrm{MHz} \\
(-127 @ 1 \mathrm{GHz})\end{array}$ & N/A & $\mathrm{N} / \mathrm{A}$ \\
\hline Jitter & $<210$ fs $^{1}$ & $<300$ fs & $<400$ fs & $<250$ fs & $\mathrm{N} / \mathrm{A}$ & $700 f s$ \\
\hline Power (mW) & $\begin{array}{c}0.8+1.0^{1} @ \\
55 \mathrm{MHz}\end{array}$ & $>0.22^{2} @ 48 \mathrm{MHz}$ & $2.2^{2} @ 40 \mathrm{MHz}$ & $0.5 @ 40 \mathrm{MHz}$ & $\mathrm{N} / \mathrm{A}$ & N/A \\
\hline CMOS Tech. & $65 \mathrm{~nm}$ & $65 \mathrm{~nm}$ & $65 \mathrm{~nm}$ & $28 \mathrm{~nm}$ & $90 \mathrm{~nm}$ & $0.18 \mathrm{um}$ \\
\hline
\end{tabular}

${ }^{1}$ Noise and power data are measured at setting for $\sim 100$ ps delay; ${ }^{2}$ Power of only DTC core, no power-hungary low-noise buffer;

${ }^{3}$ Estimation based on fractional-spur level using analysis in [21] to show the order of magnitude of INL;

\section{Table 1 Comparison with other recent work on DTC performance}

